\title{
Asymptomatic Uncountable Urinary Bladder Stones Removal: Play the Winner
}

\author{
Hani H. Nour ${ }^{a}$ b Taha F. Mahmoud ${ }^{c} \quad$ Lemya Alzaabi $^{c}$ \\ a Urology Department, Kalba Hospital, MOHAP, Sharjah, UAE; b Urology Department, Theodor Bilharz Research \\ Institute, Giza, Egypt; ${ }^{\circ}$ Radiology Department, Kalba Hospital, MOHAP, Sharjah, UAE
}

\section{Keywords}

Cystolitholapaxy · Cystolithotomy · Open surgery · Stone · Urinary bladder

\begin{abstract}
Urinary bladder stones are a common condition in elderly males, and they are usually related to infravesical obstruction secondary to prostate enlargement. Endoscopic management of bladder stone is the gold standard treatment; yet, in some cases, return to conventional open surgery is necessary. In our article, we reported the case of a 73-yearold male patient with accidentally discovered multiple urinary bladder stones. Cystolithotomy was the treatment of choice which went uneventfully with a smooth postoperative course.

(c) 2020 The Author(s)

Published by S. Karger AG, Basel
\end{abstract}

\section{Introduction}

Urinary stones are the third most common pathology affecting the urinary tract. They are exceeded only by urinary tract infections and pathologic conditions of the prostate [1].

Stone disease can be classified according to the anatomical site into upper urinary tract stones (kidney, ure-

\begin{tabular}{ll}
\hline karger@karger.com & (C) 2020 The Author(s) \\
www.karger.com/dmj & Published by S. Karger AG, Basel Karger \\
& This article is licensed under the Creative Commons Attribution- \\
Karger & NonCommercial-NoDerivatives 4.0 International License (CC BY- \\
NC-ND) (http://www.karger.com/Services/OpenAccessLicense). \\
Usage and distribution for commercial purposes as well as any dis- \\
tribution of modified material requires written permission.
\end{tabular}

ter) and lower tract stones (vesical, urethral, prostatic); lower urinary tract stones are less common, with urinary bladder stones accounting for more than $90 \%$ of them, and are usually formed de novo inside the bladder [2].

Several risk factors for urinary bladder lithiasis have been studied, including the presence of bladder outlet obstruction (BOO), recurrent infection, metabolic diseases, and anatomical abnormalities; iatrogenic causes such as a migratory intrauterine device acting as a nidus for stone formation have been also reported [3].

Bladder lithiasis is more common in males, with a male:female ratio of $4: 1$. Peak incidence is seen in 2 age groups. The first age group is children in developing countries below the age of 3 years, usually secondary to dietary and metabolic abnormalities, and the second age group is men over 50 years, where bladder lithiasis is usually related to prostatic enlargement [1-4].

We present the case of a 73-year-old male with accidentally discovered uncountable urinary bladder stones without any lower urinary tract symptoms.

\section{Case Report}

A 73-year-old male patient presented to our emergency department with fever and productive cough.

The patient has essential hypertension and is on antihypertensive medication. He also has chronic obstructive pulmonary dis- 

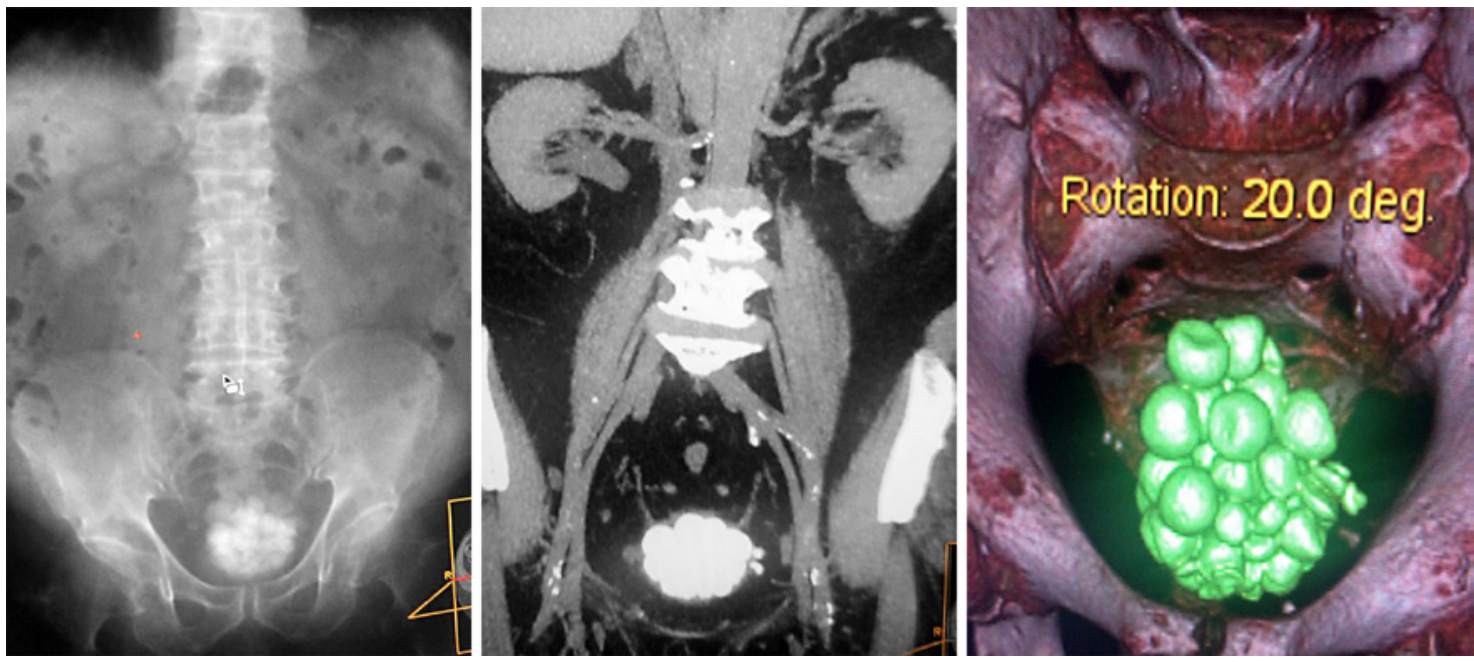

Fig. 1. Preoperative radiological findings.

ease and bronchiectasis. After the initial evaluation, he was primarily diagnosed to have an acute exacerbation of bronchiectasis and admitted to the internal medicine department.

After the initial evaluation, the biochemical study revealed elevated total leucocyte count with a shift to the left, elevated inflammatory markers, with normal renal and hepatic biochemical investigations except microscopic hematuria in routine urine analysis with no pyuria.

Forty-eight hours after admission, the patient was vitally stable, chest condition improved, and inflammatory markers began to improve; urology consultation was ordered by the internal medicine physician to evaluate hematuria.

On evaluation, the patient denied any urinary symptoms, he confirmed having an adequate urinary stream, sometimes interrupted but relating this interruption to low fluid intake. He reported waking up once at night and having no pain during urination; he did not see blood in the urine either.

When his symptoms were objectively analyzed using the International Prostate Symptoms Score (IPSS), he had a score of 18, yet he described the symptoms as moderately bothersome, and that he was not even aware of them.

On physical examination, the patient was lying comfortably in bed, his body mass index (BMI) was 36; no palpable abdominopelvic masses were noted, yet there was diffuse dullness on percussion of the suprapubic area. Digital rectal examination showed hugely enlarged prostate, firm with a smooth surface.

The complete blood count was normal together with renal function; the urine culture ordered on admission returned negative; however, his PSA was high $(11.2 \mathrm{ng} / \mathrm{dL})$.

Ultrasound examination of the urinary tract showed bilateral increased renal echogenicity, thickened urinary bladder wall, with multiple urinary bladder stones variable in size.

The prostate was estimated at around $140 \mathrm{~mL}$, while post-voiding residual urine could not be assessed due to inadequate filling.

A plain CT of the urinary tract revealed that both kidneys were normal in size and shape with no evidence of renal or ureteric stones, yet the urinary bladder showed an uncountable number of stones of different sizes; the largest that could be measured was 24 $\mathrm{mm}$ in the maximum diameter. The prostate gland was estimated to be around $170 \mathrm{~mL}$ (Fig. 1).

Uroflowmetry was done; the patient voided $184 \mathrm{~mL}$, with a maximum flow rate $(\mathrm{Q} \max )$ of $7.9 \mathrm{~mL} / \mathrm{s}$; the average flow was 6.5 $\mathrm{mL} / \mathrm{s}$, and the flow pattern was polyphasic.

After discussing the risk/benefit with the patient and his family, a decision for cystolithotomy preceded by diagnostic cystoscopy was taken. Given the large uncountable number of the stones, we decided this as the safest and fastest treatment strategy. The patient did not agree to undergo operation for prostate enlargement, and he preferred a watchful waiting protocol with a possibility of having holmium enucleation of the prostate later on.

Combined spinal-epidural anesthesia was given. Formal diagnostic cystoscopy was done and showed hugely enlarged bilobar prostate with high riding bladder neck; surprisingly, no middle lobe enlargement was present. The bladder entry by the cystoscope was difficult due to multiple stones. Inspection of the urinary bladder wall revealed no intravesical masses, yet ureteric orifices were not seen.

In the supine position, a suprapubic transverse incision of the skin, subcutaneous tissue, and rectus sheath was done. Both recti were separated with the opening of the retropubic space of Retzius; the urinary bladder was freed from the surrounding attachments and opened between two stay sutures (Fig. 2). Multiple bladder stones protruded once the bladder was opened, and the stones were removed using both straight and curved stone-grasping forceps.

A total of 89 stones of different sizes were extracted and doublechecked for the count by the circulating nurse (Fig. 3). Inspection of the bladder wall, with visualization of both ureteric orifices, was done.

The urinary bladder was closed in a double layer over a urinary catheter and a drain in the Retzius space. The postoperative course was smooth, the drain was removed at postoperative day 3 . The patient was discharged on day 4 while the urethral catheter was kept for 1 week. Before discharge, the patient was prescribed tamsulosin $0.4 \mathrm{mg}$ together with dutasteride $5 \mathrm{mg}$ per oral daily as a combined treatment for his benign prostatic hyperplasia $(\mathrm{BPH})$. 

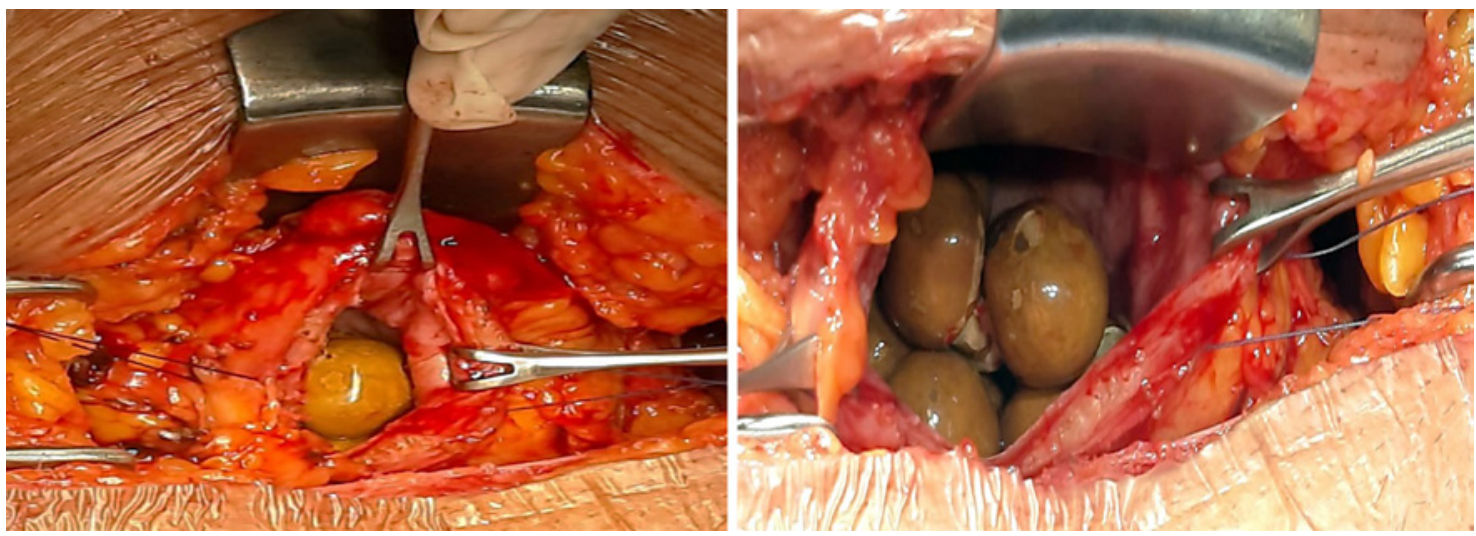

Fig. 2. Intraoperative stone extraction.
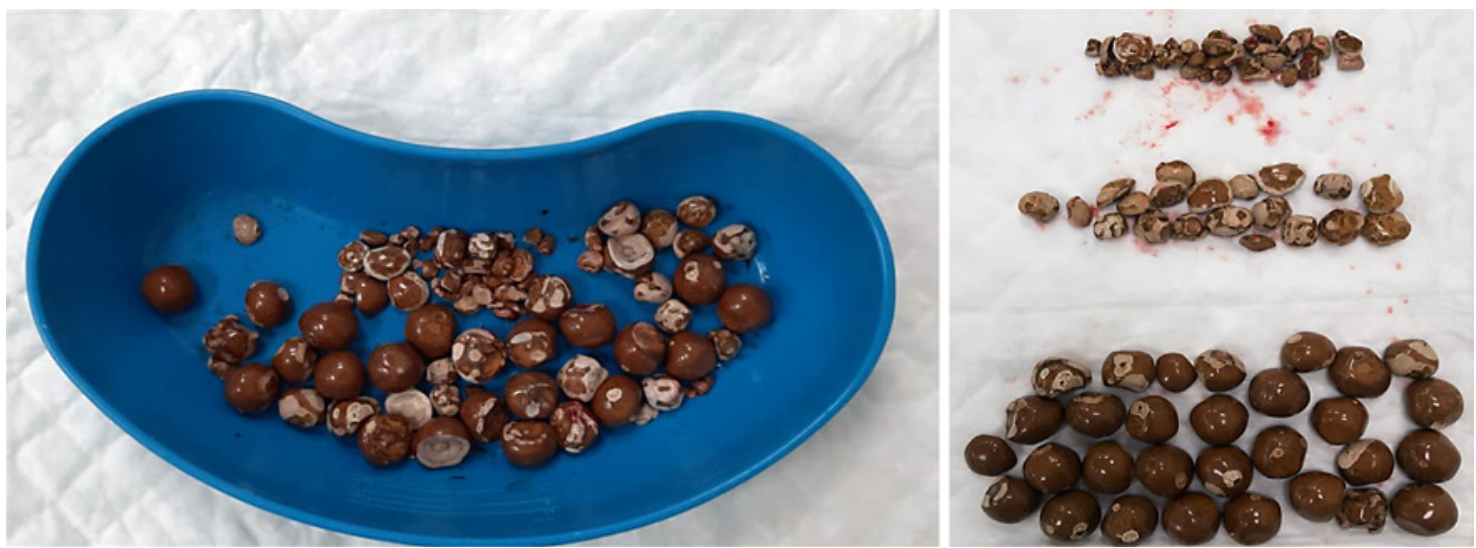

Fig. 3. Extracted stones.

At 1 week, the patient was seen for catheter removal. After removal, the patient resumed normal micturition with a relatively high postvoiding residual urine $(110 \mathrm{~mL})$. The patient was instructed to repeat uroflowmetry with postvoiding residual urine estimation 1 week later. Surgical clips were removed at day 10 postoperatively. The biochemical stone analysis showed a mixed calcium stone.

On the 15th day postoperatively, uroflowmetry was done revealing a total voided volume of $240 \mathrm{~mL}$, maximum flow rate $(\mathrm{Q}$ $\max )$ of $8.6 \mathrm{~mL} / \mathrm{s}$, with an average flow of $6.3 \mathrm{~mL} / \mathrm{s}$. At follow-up, a formal ultrasound scan of the urinary bladder showed a postvoiding residual urine of 35 and $43 \mathrm{~mL}$ on 2 different scans, respectively. The PSA level was $11.7 \mathrm{ng} / \mathrm{dL}$.

\section{Discussion}

Bladder calculi are some of the most common urolithiases in men over 50 years old. This correlates with the development of $\mathrm{BPH}$. BOO secondary to $\mathrm{BPH}$ increases the risk of chronic urinary retention, and it was postulated that the increase in the residual urine will lead to deposition of urinary crystals within the bladder predisposing to stone formation, yet this argument was debated in several studies [3]. Bladder stones secondary to BPH were also related to recurrent infection; however, unlike studies of upper urinary tract stone disease, factors contributing to the pathogenesis of bladder calculi have not been well explored [5].

Urinary bladder stones usually present with frequency, urgency, hematuria, and suprapubic discomfort. In patients with moderate to severe lower urinary tract symptoms secondary to BOO, symptoms may be overlapping; however, asymptomatic bladder calculi were observed in up to $15 \%$ of the patients [6].

In our report, the patient had no specific urinary complaint and was evaluated secondary to an accidentally discovered hematuria during workup for fever; however, the patient had an IPSS of 18 (moderate symptoms). This can be related to concurrent comorbidities and their complications distracting patient's attention. 
The main goal of stone treatment is to completely remove them, eliminate urinary obstruction, and eradicate urinary infection. Endoscopic stone removal via a transurethral approach or a percutaneous one is the standard treatment for bladder stone; conventional surgery is usually reserved for patients with complex anatomy or bladder substitution. In our case, endoscopic removal of all stones was estimated to be time-consuming, morbid, and carries high chances of conversion to open surgery. Cystolithotomy appeared to be the best option. It is quick (<45 min), safe (has a low incidence of morbidity), and effective allowing removal of all stones at the same time with no residual fragments or waiting for gravels to pass; we opted to play the winner safely in this case. The postoperative course was smooth; the patient was discharged on postoperative day 4 and was catheter free at 1 week [7].

The patient had sterile urine preoperatively and was under antibiotic coverage for his chest condition; no postoperative antibiotics were used. The patient's right to accept or decline treatment was taken into consideration; the patient refused to undergo operation for prostate after the potential risks had been clearly explained to him. Medical treatment including a combination of alpha-blockers and 5-alpha-reductase inhibitors for BPH was started preoperatively. On postoperative evaluation, the patient showed an adequate response to medication, and a follow-up protocol was initiated [8].

The patient had elevated PSA on initial assessment, but there was no previous record for his PSA. Follow-up PSA 1 month after the initial results revealed almost no change. PSA density was calculated to be $0.07 \mathrm{ng} / \mathrm{mL}$; the potential risk/benefits of the prostate biopsy were discussed with the patient [9], and he agreed to PSA monitoring.

\section{Conclusion}

Urinary bladder lithiasis is common in men with $\mathrm{BOO}$. To our knowledge, this case represents one of the largest numbers of urinary bladder stones reported. When choosing a strategy for stone removal, open surgery still has a role especially in large and multiple stones with low morbidity.

\section{Acknowledgement}

The author acknowledges the help of MOHAP and UAE administration in Kalba Hospital for their interest in starting scientific activities.

\section{Statement of Ethics}

This article follows the tenets of the World Medical Association Declaration of Helsinki guidelines for human studies. Consent was taken from the patient to share the case.

\section{Conflict of Interest Statement}

The authors have no conflicts of interest to declare.

\section{Funding Sources}

None.

\section{Author Contributions}

H.H.N. was responsible for collecting the data, writing the manuscript, and submitting it. L.A. suggested to publish the case, obtained necessary administrative approvals, and helped to revise the final manuscript. T.F.M. was responsible for preparing the radiological material and editing the figures.

\section{References}

1 Sorokin I, Mamoulakis C, Miyazawa K, Rodgers A, Talati J, Lotan Y. Epidemiology of stone disease across the world. World J Urol. 2017 Sep;35(9):1301-20.

2 Trinchieri A. Epidemiology of urolithiasis: an update. Clin Cases Miner Bone Metab. 2008 May;5(2):101-6.

3 Childs MA, Mynderse LA, Rangel LJ, Wilson TM, Lingeman JE, Krambeck AE. Pathogenesis of bladder calculi in the presence of urinary stasis. J Urol. 2013 Apr; 189(4):1347-51.

4 Huang W, Cao JJ, Cao M, Wu HS, Yang YY, Xu ZM, et al. Risk factors for bladder calculi in patients with benign prostatic hyperplasia.
Medicine (Baltimore). 2017 Aug;96(32): e7728.

5 Yoo TK, Cho HJ. Benign prostatic hyperplasia: from bench to clinic. Korean J Urol. 2012 Mar;53(3):139-48.

6 Jung JH, Park J, Kim WT, Kim HW, Kim HJ, Hong $S$, et al. The association of benign prostatic hyperplasia with lower urinary tract stones in adult men: A retrospective multicenter study. Asian J Urol. 2018 Apr;5(2):118-21.

7 Habib E, Ayman LM, ElSheemy MS, El-Feel AS, Elkhouly A, Nour HH, et al. Holmium Laser Enucleation vs Bipolar Plasmakinetic Enucleation of a Large Volume Benign Pros- tatic Hyperplasia: A Randomized Controlled Trial. J Endourol. 2020 Mar;34(3):330-8.

8 Gratzke C, Bachmann A, Descazeaud A, Drake MJ, Madersbacher S, Mamoulakis C, et al. EAU Guidelines on the assessment of nonneurogenic male lower urinary tract symptoms including benign prostatic obstruction. Eur Urol. 2015 Jun;67(6):1099-109.

9 Mottet N, Bellmunt J, Bolla M, Briers E, Cumberbatch MG, De Santis M, et al. EAU-ESTRO-SIOG Guidelines on Prostate Cancer. Part 1: Screening, Diagnosis, and Local Treatment with Curative Intent. Eur Urol. 2017 Apr;71(4):618-29. 